\title{
A NEW TWO-PARAMETER DISTRIBUTION WITH DECREASING, INCREASING, BATHTUB HAZARD RATE FUNCTIONS AND ITS APPLICATIONS
}

\author{
F. Alqallaf, M.E. Ghitany ${ }^{\S}$, M.F. Alfahad \\ Department of Statistics and Operations Research \\ Faculty of Science, Kuwait University \\ Safat 13060, KUWAIT
}

\begin{abstract}
In the literature, two-parameter distributions which exhibit all three types of decreasing, increasing and bathtub shape hazard rate functions are very few. In this paper, we propose a new two-parameter distribution, called Gompertz-weighted exponential distribution, having these three types of hazard rate functions. The proposed distribution is obtained by mixing the frailty parameter of the Gompertz distribution by weighted exponential distribution. The parameters are estimated by the maximum likelihood method and their performance is examined by extensive simulation studies. Three real data applications are provided to illustrate the superiority of the proposed distribution over many well known two-parameter distributions.
\end{abstract}

AMS Subject Classification: 62F10, 62F15, 62F 25

Key Words: mixture; bathtub hazard rate; mean residual life; order statistics; stochastic orders

\section{Introduction}

The hazard rate function is an important quantity characterizing life phenomena. Many known distributions in statistics have monotone (dereasing/increasing) hazard rate functions, for example, gamma, Weibull, exponentiated exponential, Lomax, linea failure rate distributions. Monotone hazard rate functions

Received: March 30, 2020

(c) 2020 Academic Publications

${ }^{\S}$ Correspondence author 
are found to be popular among many reliability engineers. However, it is widely believed that many electronic items such as silicon integrated circuits, exhibit bathtub shape hazard rate function. This belief is supported by data sets in many industries. Comprehensive reviews of the known distributions that exhibit bathtub shape hazard rate functions are provided in [1], [2] and [3]. These distributions can have many parameters which complicate their analysis and estimation process.

In this paper, we are interested in two-parameter distributions which exhibit bathtub shape hazard rate functions, see, for example, Burr X [4], exponential power [5], double exponential power [6], Chen [7], weighted Lindley [8], generalized Lindley [9]. To the best of our knowledge, generalized Lindley distribution is the only two-parameter distribution which exhibits bathtub shape as well as monotone decreasing and monotone increasing hazard rate functions. Generalized Lindley distribution is obtained by exponentiated the distribution function of Lindley distribution.

In this paper we propose a new two-parameter distribution which exhibits bathtub shape as well as monotone decreasing and monotone increasing hazard rate functions. The proposed distribution is obtained by mixing the frailty parameter of the Gompertz distribution by a one-parameter weighted exponential distribution. Structural properties of the new distribution as well as estimation, simulation nd applications are presented.

The contents of the paper are organized as follows: Section 2 contains the model derivation, its structural properties including the cumulative distribution, the quantile, the hazard rate and the mean residual life functions and their properties. Section 3 contains the order statistics of the proposed model and their limiting distributions. The stochastic orders are shown in Section 4. The maximum likelihood estimation of the parameters and simulation studies to examine the performance of the estimates are given in Section 5. Three applications of real data sets are provided in Section 6 to demonstrate the superiority of the proposed model over many well known two-parameter models.

\section{The model and structural properties}

\subsection{Probability density function}

Let $X \mid \Theta$ be a conditional random variable having the Gompertz distribution with frailty parameter $\theta$ and scale parameter $\lambda$, with conditional probability density function (p.d.f.):

$$
f(x \mid \theta)=\theta \lambda \exp \left[\lambda x-\theta\left(e^{\lambda x}-1\right)\right], \quad x>0, \quad \theta, \lambda>0 .
$$


Assume that $\Theta$ has a weighted exponential distribution with parameter $\alpha$ and p.d.f.:

$$
g(\theta)=2 \alpha e^{-\alpha \theta}\left(1-e^{-\alpha \theta}\right), \quad \theta>0, \quad \alpha>0 .
$$

The unconditional p.d.f. of $X$ is given by

$$
\begin{aligned}
f(x) & =\int_{0}^{\infty} f(x \mid \theta) \cdot g(\theta) d \theta \\
& =2 \alpha \lambda e^{\lambda x}\left[\frac{1}{\left(e^{\lambda x}+\alpha-1\right)^{2}}-\frac{1}{\left(e^{\lambda x}+2 \alpha-1\right)^{2}}\right] \\
& =\frac{2 \alpha^{2} \lambda e^{\lambda x}\left(2 e^{\lambda x}+3 \alpha-2\right)}{\left(e^{\lambda x}+\alpha-1\right)^{2}\left(e^{\lambda x}+2 \alpha-1\right)^{2}}, \quad x>0, \quad \alpha, \lambda>0 .
\end{aligned}
$$

The distribution with p.d.f. (1) will be denoted by $\operatorname{GoWE}(\alpha, \lambda)$.

Note that $f(x)$ can be expressed as a two-parameter negative mixture with p.d.f.:

$$
f(x)=2 f_{1}(x)-f_{2}(x), \quad x>0,
$$

where, for $j=1,2$,

$$
f_{j}(x)=\frac{j \alpha \lambda e^{-\lambda x}}{\left[1-(1-j \alpha) e^{-\lambda x}\right]^{2}}, \quad x>0, \quad \alpha, \lambda>0,
$$

is the p.d.f. of Marshal-Olkin extended exponential distribution, see [10].

Theorem 1. For all $\lambda>0, f(x)$ is decreasing (unimodal) if $\alpha \leq \frac{7}{3}$ (if $\left.\alpha>\frac{7}{3}\right)$ with

$$
f(0)=\frac{3 \lambda}{2 \alpha}, \quad f(\infty)=0 .
$$

Proof. The first derivative of $f(x)$ can be expressed as

$$
f^{\prime}(x)=\frac{\alpha^{2}(\alpha+1) \lambda^{2} e^{\lambda x}}{\left(e^{\lambda x}+\alpha-1\right)^{3}\left(e^{\lambda x}+2 \alpha-1\right)^{3}} \xi\left(e^{\lambda x}\right),
$$

where, for $z=e^{\lambda x}>1$,

$$
\xi(z)=-4 z^{3}-3(3 \alpha-2) z^{2}-\alpha^{2} z+(\alpha-1)(2 \alpha-1)(3 \alpha-2),
$$

with $\xi(1)=2 \alpha^{2}(3 \alpha-7)$ and $\xi(\infty)=-\infty$.

The cubic function $\xi(z)$ is decreasing in $z$. Therefore, $\xi(z)$ is negative (changes sign from positive to negative) if $\xi(1) \leq 0(\xi(1)>0)$. Since $f^{\prime}(x)$ has the same sign as $\xi(z)$, the theorem follows. 
Figure 1 shows the p.d.f. of the GoWE distribution for selected values of $\alpha$ and $\lambda=1$.
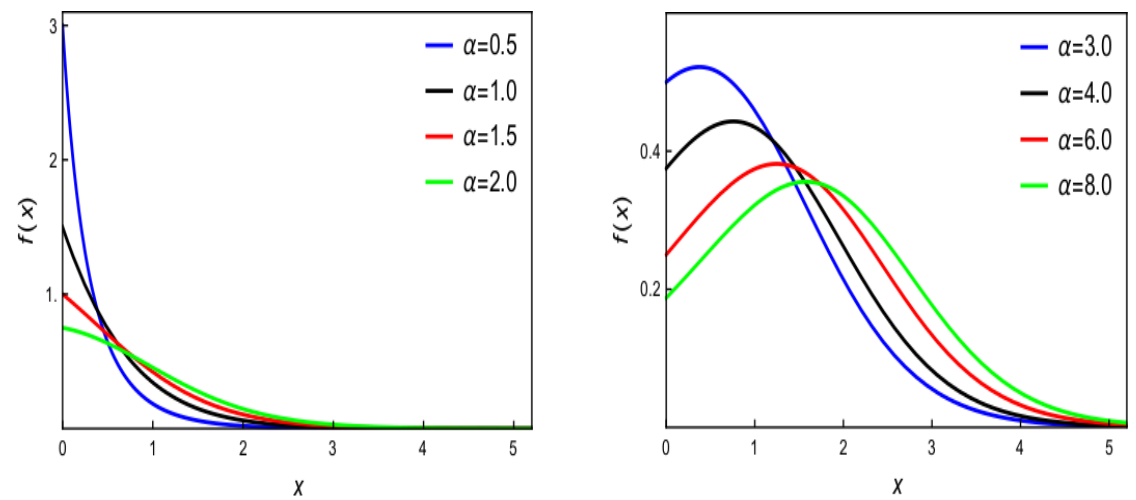

Figure 1: The p.d.f. of the GoWE distribution for selected values of $\alpha$ and $\lambda=1$.

\subsection{Cumulative distribution and quantile functions}

The cumulative distribution function (c.d.f.) of the $\operatorname{GoWE}(\alpha, \lambda)$ distribution is given by

$$
\begin{aligned}
F(x) & =P(X \leq x) \\
& =1-\frac{2 \alpha^{2}}{\left(e^{\lambda x}-1+\alpha\right)\left(e^{\lambda x}-1+2 \alpha\right)}, x>0, \alpha, \lambda>0 .
\end{aligned}
$$

The $q$ th quantile $x_{q}$ of the $\operatorname{GoWE}(\alpha, \lambda)$ distribution is the solution of $F\left(x_{q}\right)=q$, where $0<q<1$. That is, the solution of

$$
z_{q}^{2}+(3 \alpha-2) z_{q}+(\alpha-1)(2 \alpha-1)-\frac{2 \alpha^{2}}{1-q}=0, \quad z_{q}=e^{\lambda x_{q}}>1 .
$$

The quadratic equation (3) has a unique real solution

$$
z_{q}=1+\frac{\alpha}{2}\left(\sqrt{\frac{8}{1-q}+1}-3\right)
$$

that is

$$
x_{q}=F^{-1}(q)=\frac{1}{\lambda} \ln \left[1+\frac{\alpha}{2}\left(\sqrt{\frac{8}{1-q}+1}-3\right)\right] \text {. }
$$


This quantile function is increasing in $\alpha$ and decreasing in $\lambda$.

Figure 2 shows the c.d.f. and quantile function of the GoWE distribution for selected values of $\alpha$ and $\lambda=1$.
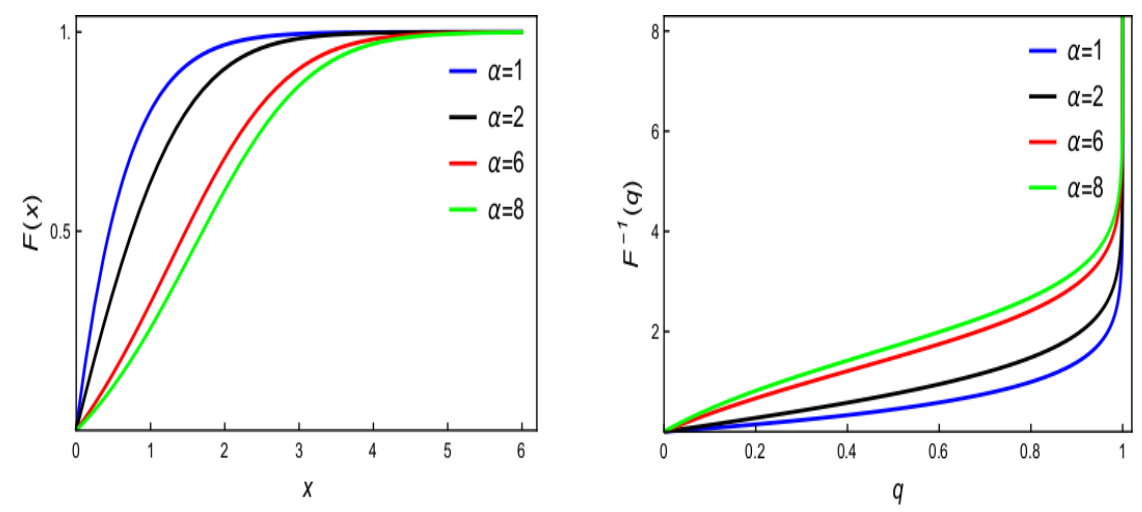

Figure 2: The c.d.f. and quantile function of the GoWE distribution for selected values of $\alpha$ and $\lambda=1$.

\section{Remarks:}

(i) The quantile function is useful in generating random data from the $\operatorname{GoWE}(\alpha, \lambda)$ distribution.

(ii) The quartiles of the $\operatorname{GoWE}(\alpha, \lambda)$ distribution are given by $x_{0.25}, x_{0.50}, x_{0.75}$, respectively.

\subsection{Hazard rate function}

The hazard rate function (h.r.f.) of the $\operatorname{GoWE}(\alpha, \lambda)$ distribution is given by

$$
\begin{aligned}
h(x) & =\frac{f(x)}{1-F(x)} \\
& =\frac{\lambda e^{\lambda x}\left(2 e^{\lambda x}+3 \alpha-2\right)}{\left(e^{\lambda x}+\alpha-1\right)\left(e^{\lambda x}+2 \alpha-1\right)}, \quad x>0, \quad \alpha, \lambda>0 .
\end{aligned}
$$

The following theorem shows the possible shapes of the h.r.f. of the $\operatorname{GoWE}(\alpha, \lambda)$ distribution.

Theorem 2. For all $\lambda>0, h(x)$ is 
(i) decreasing if $\alpha \leq \frac{2}{3}$,

(ii) bathtub if $\frac{2}{3}<\alpha<\frac{5}{6}$,

(iii) increasing if $\alpha \geq \frac{5}{6}$,

with

$$
h(0)=f(0)=\frac{3 \lambda}{2 \alpha}, \quad h(\infty)=2 \lambda .
$$

Proof. The first derivative of $h(x)$ can be expressed as

$$
h^{\prime}(x)=\frac{\lambda^{2} e^{\lambda x}}{\left(e^{\lambda x}+\alpha-1\right)^{2}\left(e^{\lambda x}+2 \alpha-1\right)^{2}} \eta\left(e^{\lambda x}\right),
$$

where

$$
\eta(z)=(3 \alpha-2) z^{2}+4(\alpha-1)(2 \alpha-1) z+(\alpha-1)(2 \alpha-1)(3 \alpha-2),
$$

and $z=e^{\lambda x}>1$. Note that

$$
\eta(1)=\alpha^{2}(6 \alpha-5), \quad \eta(\infty)= \begin{cases}-\infty, & \text { for } \alpha \leq \frac{2}{3} \\ \infty, & \text { for } \alpha>\frac{2}{3}\end{cases}
$$

For $\alpha \leq \frac{2}{3}<\frac{5}{6}<0, \eta(z)$ is decreasing in $z$ and hence $\eta(z)$ is negative.

For $\frac{2}{3}<\alpha<\frac{5}{6}$, the function $\eta(z)$ has an absolute minimum at the point $z_{1}=\frac{2(1-\alpha)(2 \alpha-1)}{3 \alpha-2}>1$ and hence $\eta(z)$ changes sign from negative to positive.

For $\alpha \geq \frac{5}{6}$, is increasing in $z$ and hence $\eta(z)$ is positive.

Since $h^{\prime}(x)$ has the same sign as $\eta(z)$, the theorem follows.

Figure 3 shows the h.r.f. of the GoWE distribution for selected values of $\alpha$ and $\lambda=1$.

\subsection{Mean residual life function}

An alternative ageing measure widely used in applications is the mean residual life function (m.r.l.f.), defined as

$$
\mu(x)=E(X-x \mid X>x)=\frac{1}{1-F(x)} \int_{x}^{\infty} 1-F(y) d y, \quad x>0 .
$$

Evidently $\mu(0)=E(X)=\mu$ is the mean of $X$. 

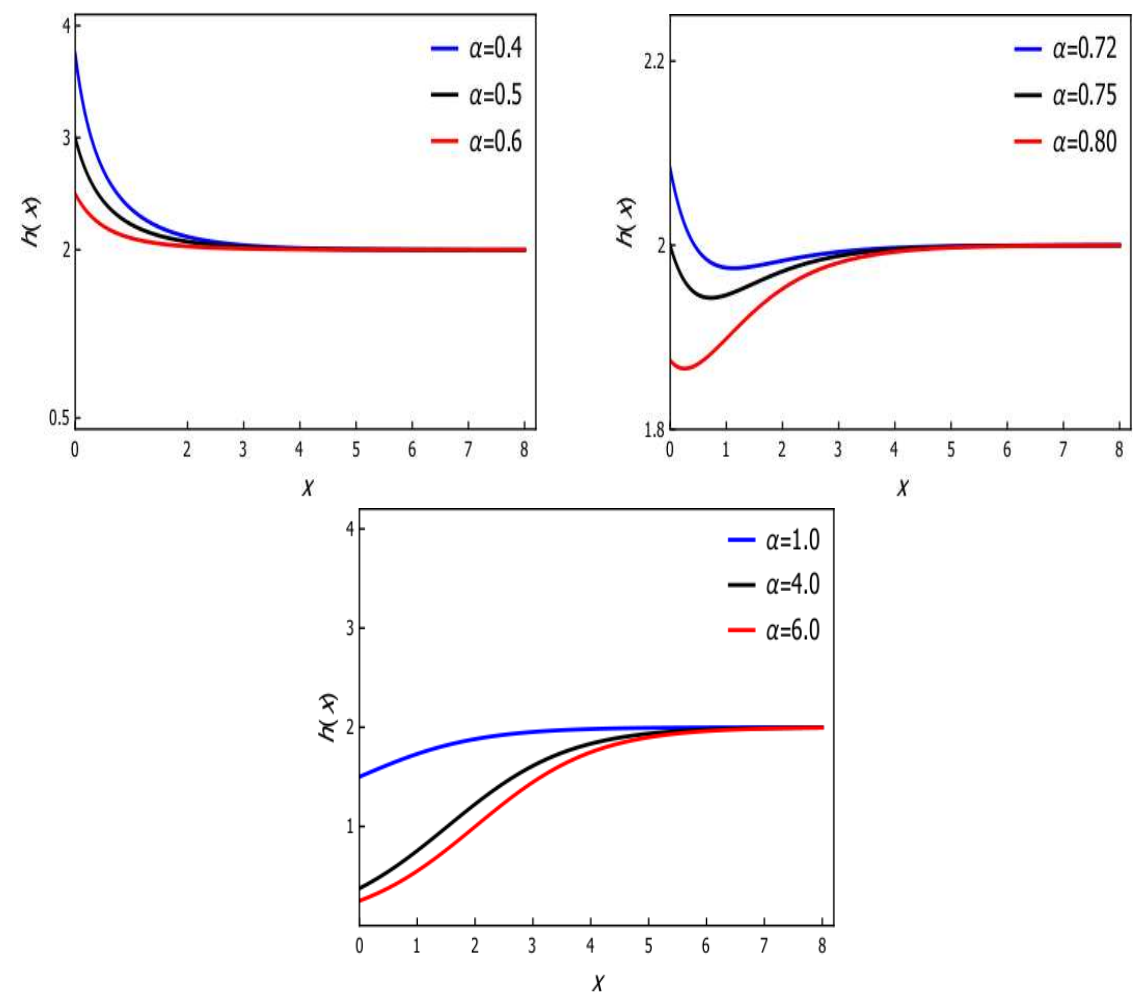

Figure 3: The h.r.f. of the GoWE distribution for selected values of $\alpha$ and $\lambda=1$.

It is well known that decreasing (increasing) h.r.f. implies increasing (decreasing) m.r.l.f., see [11]. On the other hand, a bathtub hazard rate function may produce an upside-down bathtub (decreasing) if $f(0) \mu>1(f(0) \mu \leq 1)$, see [18].

The following theorem shows the possible shapes of the MRLF of the $\operatorname{GoWE}(\alpha, \lambda)$ distribution.

Theorem 3. For all $\lambda>0, \mu(x)$ is

(i) increasing if $0<\alpha \leq \frac{2}{3}$;

(ii) upside-down bathtub if $\frac{2}{3}<\alpha<\alpha_{1}=0.793379$;

(iii) decreasing if $\alpha \geq \alpha_{1}=0.793379$, with

$$
\mu(0)=\mu=\frac{2 \alpha}{\lambda}\left[-\frac{\ln (\alpha)}{1-\alpha}+\frac{\ln (2 \alpha)}{1-2 \alpha}\right], \quad \mu(\infty)=\frac{1}{2 \lambda} .
$$


Proof. We have shown in Theorem 2, that for all $\lambda>0$ and $\alpha \leq \frac{2}{3}\left(\alpha \geq \frac{5}{6}=\right.$ $0.8333), h(x)$ is decreasing (increasing) and hence, by $[11], \mu(x)$ is increasing (decreasing).

Also, we have shown in Theorem 2, that for all $\lambda>0$ and $\frac{2}{3}<\alpha<\frac{5}{6}, h(x)$ is bathtub. Since

$$
f(0) \mu=3\left[-\frac{\ln (\alpha)}{1-\alpha}+\frac{\ln (2 \alpha)}{1-2 \alpha}\right], \quad \frac{2}{3}<\alpha<\frac{5}{6},
$$

it follows that $f(0) \mu>1(\leq 1)$ if $\frac{2}{3}<\alpha<\alpha_{1} \leq \frac{5}{6}\left(\frac{2}{3}<\alpha_{1} \leq \alpha<\frac{5}{6}\right)$, where $\alpha_{1}$ is such that

$$
3\left[-\frac{\ln \left(\alpha_{1}\right)}{1-\alpha_{1}}+\frac{\ln \left(2 \alpha_{1}\right)}{1-2 \alpha_{1}}\right]=1, \quad \frac{2}{3}<\alpha_{1}<\frac{5}{6} .
$$

A numerical solution of the last equation gives $\alpha_{1}=0.793379$. Therefore, by [18], $\mu(x)$ is upside-down bathtub (decreasing) if $\frac{2}{3}<\alpha<\alpha_{1}\left(\alpha_{1} \leq \alpha<\frac{5}{6}\right)$.

Figure 4 shows the the m.r.l.f. of the GoWE distribution for selected values of $\alpha$ and $\lambda=1$.

\subsection{Moments and associated measures}

The $r$ th raw moment (about the origin) of the GoWE distribution is given by

$$
\begin{aligned}
\mu_{r}^{\prime} & =E\left(X^{r}\right) \\
& =\frac{2 r \alpha}{\lambda^{r}} \int_{0}^{\infty} t^{r-1}\left[\frac{1}{e^{t}-(1-\alpha)}-\frac{1}{e^{t}-(1-2 \alpha)}\right] d t \\
& =\frac{2 r ! \alpha}{\lambda^{r}}\left[\frac{\operatorname{Li}_{r}(1-\alpha)}{1-\alpha}-\frac{\operatorname{Li}_{r}(1-2 \alpha)}{1-2 \alpha}\right],
\end{aligned}
$$

where

$$
\operatorname{Li}_{r}(z)=\frac{z}{\Gamma(r)} \int_{0}^{\infty} \frac{t^{r-1}}{e^{t}-z} d t, \quad r>0, \quad-\infty<z<1,
$$

is the polylogarithm function. In particular, $\operatorname{Li}_{1}(z)=-\ln (1-z)$.

The polylogarithm function is thoroughly covered in the book by [13]. It can be evaluated to arbitrary numerical precision using, for example, in Mathematica package using the function PolyLog $[r, z]$. 

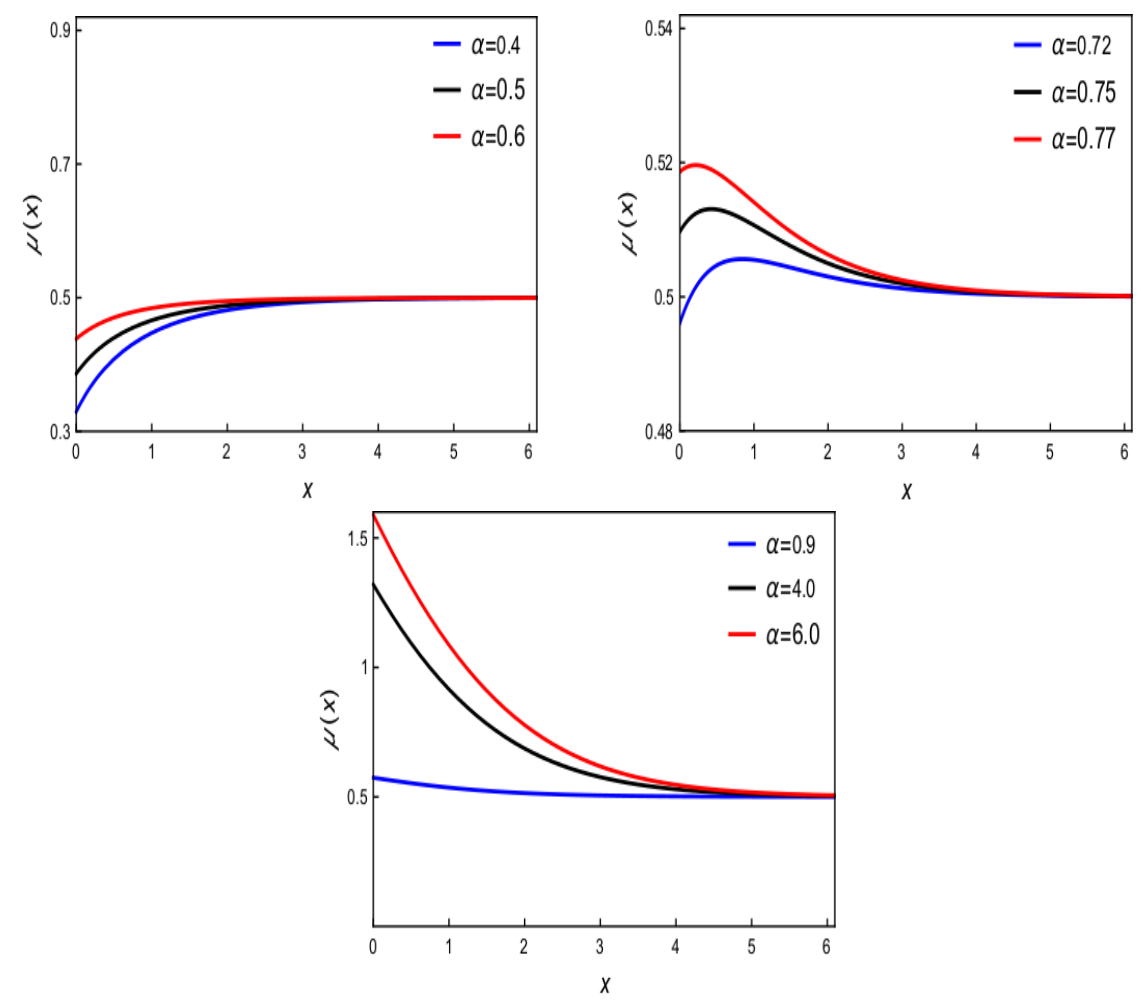

Figure 4: The m.r.l.f. of the GoWE distribution for selected values of $\alpha$ and $\lambda=1$.

The first four raw moments are

$$
\begin{aligned}
\mu & =\frac{2 \alpha}{\lambda}\left[-\frac{\ln (\alpha)}{1-\alpha}+\frac{\ln (2 \alpha)}{1-2 \alpha}\right], \\
\mu_{2}^{\prime} & =\frac{4 \alpha}{\lambda^{2}}\left[\frac{\mathrm{Li}_{2}(1-\alpha)}{1-\alpha}-\frac{\mathrm{Li}_{2}(1-2 \alpha)}{1-2 \alpha}\right], \\
\mu_{3}^{\prime} & =\frac{12 \alpha}{\lambda^{3}}\left[\frac{\mathrm{Li}_{3}(1-\alpha)}{1-\alpha}-\frac{\mathrm{Li}_{3}(1-2 \alpha)}{1-2 \alpha}\right], \\
\mu_{4}^{\prime} & =\frac{48 \alpha}{\lambda^{4}}\left[\frac{\mathrm{Li}_{4}(1-\alpha)}{1-\alpha}-\frac{\mathrm{Li}_{4}(1-2 \alpha)}{1-2 \alpha}\right] .
\end{aligned}
$$

The variance, skewness and kurtosis of the GoWE distribution are obtained 
from the expressions

$$
\begin{aligned}
\sigma^{2} & =E(X-\mu)^{2}=\mu_{2}^{\prime}-\mu^{2}, \\
\text { Skewness } & =\frac{E(X-\mu)^{3}}{\sigma^{3}}=\frac{\mu_{3}^{\prime}-3 \mu_{2}^{\prime} \mu+2 \mu^{3}}{\sigma^{3}}, \\
\text { Kurtosis } & =\frac{E(X-\mu)^{4}}{\sigma^{4}}=\frac{\mu_{4}^{\prime}-4 \mu_{3}^{\prime} \mu+6 \mu_{2}^{\prime} \mu^{2}-3 \mu^{4}}{\sigma^{4}},
\end{aligned}
$$

upon substituting for the raw moments.

Note that the skewness and kurtosis are independent of the scale parameter $\lambda$ and depend only on the parameter $\alpha$.

Figure 5 (6) shows the skewness (kurtosis) of the GoWE distribution as a function of $\alpha$.

Figure 6 shows that the skewness may be positive/negative which can be useful in modelling skewed to the right/left data sets.
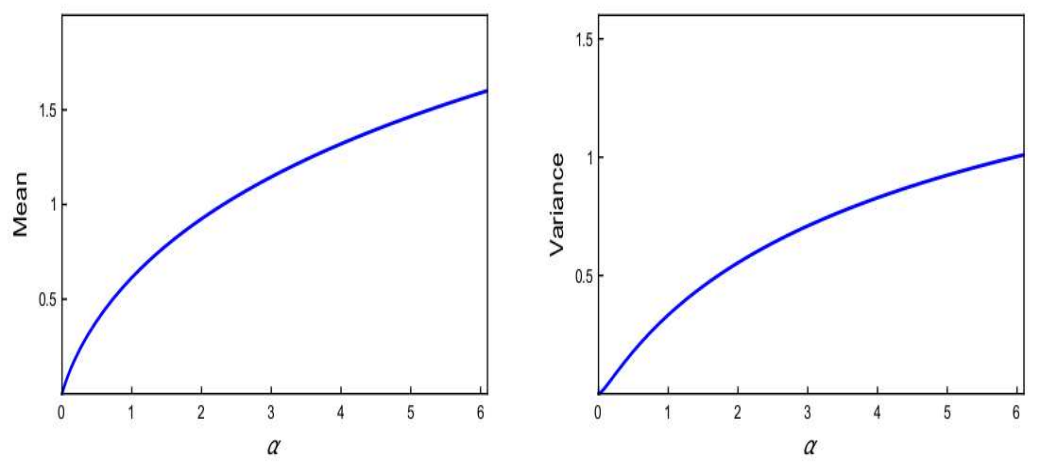

Figure 5: Mean and variance of the GoWE distribution as a function of $\alpha$ and $\lambda=1$.

\section{Order statistics}

Let $X_{1}, X_{2}, \ldots, X_{n}$ be a random sample from a distribution with p.d.f. $f(x)$, c.d.f. $F(x)$ and let $X_{i: n}$ denote the $i$ th order statistic. The p.d.f. of the $i$ th order statistic $X_{i: n}$ is given by (see [14]).

$$
f_{i: n}(x)=n\left(\begin{array}{c}
n-1 \\
i-1
\end{array}\right)[F(x)]^{i-1}[1-F(x)]^{n-i} f(x) .
$$



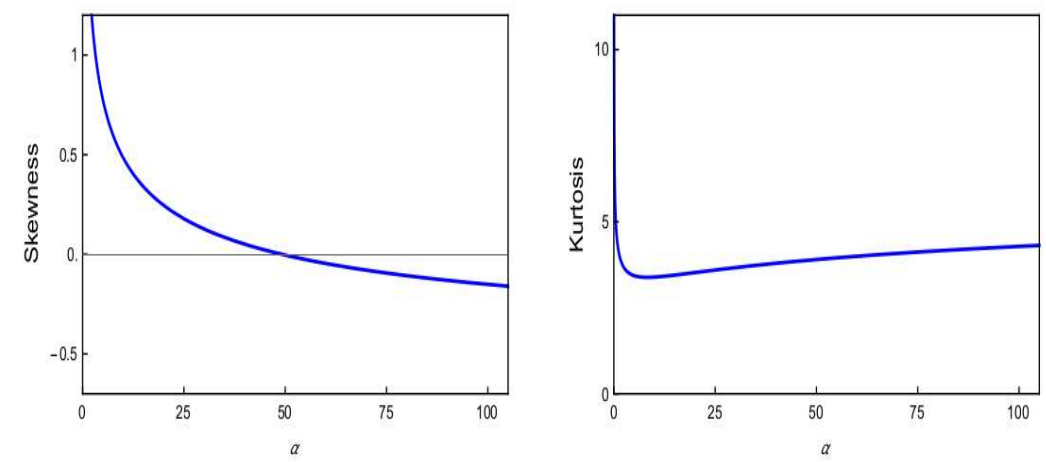

Figure 6: Skewness and kurtosis of the GoWE distribution as a function of $\alpha$.

In particular, the p.d.f. of the minimum order statistic $X_{1: n}$ and maximum order statistic $X_{n: n}$, respectively, are given by

$$
\begin{aligned}
& f_{1: n}(x)=n[1-F(x)]^{n-1} f(x), \\
& f_{n: n}(x)=n[F(x)]^{n-1} f(x) .
\end{aligned}
$$

In the following theorem, we provide the limiting distributions of $X_{1: n}$ and $X_{n: n}$ arising from the GoWE distribution.

Theorem 4. Let $X_{1: n}$ and $X_{n: n}$ be the minimum and maximum of a random sample $X_{1}, X_{2}, \ldots, X_{n}$ from $\operatorname{GoWE}(\alpha, \lambda)$, respectively. Then:

(a) $\quad \lim _{n \rightarrow \infty} P\left\{\frac{X_{1: n}-a_{n}^{*}}{b_{n}^{*}} \leq x\right\}=1-e^{-x}, \quad x>0$

(b) $\quad \lim _{n \rightarrow \infty} P\left\{\frac{X_{n: n}-a_{n}}{b_{n}} \leq t\right\}=\exp \left(-e^{-t}\right), \quad-\infty<t<\infty$

where $a_{n}^{*}=0, b_{n}^{*}=F^{-1}(1 / n), a_{n}=F^{-1}(1-1 / n)$, and $b_{n}=F^{-1}(1-1 /(n e))$ $F^{-1}(1-1 / n)$.

Proof. For the GoWE $(\alpha, \lambda)$, we have, by using L'Hospital rule,

$$
\lim _{\epsilon \rightarrow 0+} \frac{F(\epsilon x)}{F(\epsilon)}=x \lim _{\epsilon \rightarrow 0+} \frac{f(\epsilon x)}{f(\epsilon)}=x, \quad x>0 .
$$


Therefore, by Theorem 8.3.6 (ii) of [14], the minimal domain of attraction of the $\operatorname{GoWE}(\alpha, \lambda)$ distribution is the standard exponential distribution, proving part (a).

For the $\operatorname{GoWE}(\alpha, \lambda)$, we have

$$
\begin{aligned}
\lim _{x \rightarrow \infty} \frac{1-F(x+\mu(x) t)}{1-F(x)} & =\lim _{x \rightarrow \infty} \frac{1-F\left(x+\frac{t}{2 \lambda}\right)}{1-F(x)} \\
& =e^{-t}, \quad-\infty<t<\infty
\end{aligned}
$$

Therefore, by Theorem 8.3.2 (iii) of [14], the maximal domain of attraction of the $\operatorname{GoWE}(\alpha, \lambda)$ distribution is the standard Gumbel (extreme value) distribution, proving part (b).

Now, we use Theorem 4 to find the limiting distribution of any order statistic.

Theorem 5. Let $X_{1: n} \leq X_{2: n} \leq \ldots \leq X_{n: n}$ be the order statistics of a random sample $X_{1}, X_{2}, \ldots, X_{n}$ from $\operatorname{GoWE}(\alpha, \lambda)$. Then, for any fixed $i \geq 1$,

$$
\begin{aligned}
& \text { (a) } \quad \lim _{n \rightarrow \infty} P\left\{\frac{X_{i: n}-a_{n}^{*}}{b_{n}^{*}} \leq x\right\}=1-\sum_{r=0}^{i-1} e^{-x} \frac{x^{r}}{r !}, \quad x>0, \\
& \text { (b) } \quad \lim _{n \rightarrow \infty} P\left\{\frac{X_{n-i+1: n}-a_{n}}{b_{n}} \leq t\right\}=\sum_{r=0}^{i-1} \exp \left(-e^{-t}\right) \frac{e^{-r t}}{r !}, \\
& -\infty<t<\infty .
\end{aligned}
$$

Proof. The theorem follows from Equations (8.4.2) and (8.4.3) of [14].

\section{Stochastic orders}

Stochastic ordering of positive continuous random variables is an important tool to judge the comparative behavior.

For two random variables $X$ and $Y$, we consider the following stochastic orders (for all $x$ ):

(i) stochastic order $\left(X \preceq_{S T} Y\right)$ if $F_{X}(x) \geq F_{Y}(x)$;

(ii) hazard rate order $\left(X \preceq_{H R} Y\right)$ if $h_{X}(x) \geq h_{Y}(x)$;

(iii) mean residual life order $\left(X \preceq_{M R L} Y\right)$ if $\mu_{X}(x) \leq \mu_{Y}(x)$; 
(iv) likelihood ratio order $\left(X \preceq_{L R} Y\right)$ if $\frac{f_{X}(x)}{f_{Y}(x)}$ decreases in $x$.

The following implications ([15]), are well known:

$$
\begin{gathered}
X \preceq_{L R} Y \quad X \preceq_{H R} Y \quad \Rightarrow \quad X \preceq_{M R L} Y \\
\Downarrow \\
X \preceq_{S T} Y
\end{gathered}
$$

For fixed scale parameter $\lambda$, the following theorem shows that GoWE distributions are ordered according to the likelihood ratio.

Theorem 6. Let $X \sim \operatorname{GoWE}\left(\alpha_{1}, \lambda\right)$ and $Y \sim \operatorname{GoWE}\left(\alpha_{2}, \lambda\right)$. If $0<\alpha_{1} \leq$ $\alpha_{2}$ and $\lambda>0$, then $X \preceq_{L R} Y$ and hence $X \preceq_{H R} Y, X \preceq_{M R L} Y, X \preceq_{S T} Y$.

Proof. First note that

$$
\frac{d}{d x} \frac{f_{X}(x)}{f_{Y}(x)}=2\left(\alpha_{1}-\alpha_{2}\right) \lambda e^{\lambda x} G\left(e^{\lambda x}-1, \alpha_{1}, \alpha_{2}\right) \frac{f_{X}(x)}{f_{Y}(x)},
$$

where, for $z=e^{\lambda x}-1>0$,

$$
\begin{aligned}
G\left(z, \alpha_{1}, \alpha_{2}\right) & =\frac{1}{\left(z+\alpha_{1}\right)\left(z+\alpha_{2}\right)}+\frac{2}{\left(z+2 \alpha_{1}\right)\left(z+2 \alpha_{2}\right)} \\
& -\frac{3}{\left(2 z+3 \alpha_{1}\right)\left(2 z+3 \alpha_{2}\right)} \\
& \geq \frac{3}{\left(z+2 \alpha_{1}\right)\left(z+2 \alpha_{2}\right)}-\frac{3}{\left(2 z+3 \alpha_{1}\right)\left(2 z+3 \alpha_{2}\right)} \\
& \geq 0 .
\end{aligned}
$$

Therefore, $\frac{d}{d x} \frac{f_{X}(x)}{f_{Y}(x)} \leq 0$, for $\alpha_{1} \leq \alpha_{2}$, i.e. $f_{X}(x) / f_{Y}(x)$ is decreasing in $x$. That is $X \preceq_{L R} Y$. The remaining statements follow from (11).

For fixed shape parameter $\alpha$, the following theorem shows that GoWE distributions are ordered according to the hazard rate.

Theorem 7. Let $X \sim \operatorname{GoWE}\left(\alpha, \lambda_{1}\right)$ and $Y \sim \operatorname{GoWE}\left(\alpha, \lambda_{2}\right)$. If $\alpha \geq 1$ and $\lambda_{1} \geq \lambda_{2}$, then $X \preceq_{H R} Y$ and hence $X \preceq_{M R L} Y, X \preceq_{S T} Y$.

Proof. Since $\alpha \geq 1$ and $\lambda_{1} \geq \lambda_{2}>0$, it follows that

$$
h_{X}(x)=\frac{\lambda_{1} e^{\lambda_{1} x}\left(2 e^{\lambda_{1} x}+3 \alpha-2\right)}{\left(e^{\lambda_{1} x}+\alpha-1\right)\left(e^{\lambda_{1} x}+2 \alpha-1\right)}
$$




$$
\begin{aligned}
& =\lambda_{1}\left(\frac{1}{1+(\alpha-1) e^{-\lambda_{1} x}}+\frac{1}{1+(2 \alpha-1) e^{-\lambda_{1} x}}\right) \\
& \geq \lambda_{2}\left(\frac{1}{1+(\alpha-1) e^{-\lambda_{2} x}}+\frac{1}{1+(2 \alpha-1) e^{-\lambda_{2} x}}\right) \\
& =h_{Y}(x) .
\end{aligned}
$$

Therefore, $X \preceq_{H R} Y$. The remaining statements follow from (11).

\section{Estimation of the parameters}

\subsection{Maximum likelihood estimation}

Let $x_{1}, x_{2}, \ldots, x_{n}$ be a random sample from the $\operatorname{GoWE}(\alpha, \lambda)$ distribution with p.d.f. (1). The log-likelihood function is given by

$$
\begin{aligned}
\ell(\alpha, \lambda)= & n \ln \left(2 \alpha^{2} \lambda\right)+n \lambda \bar{x}+\sum_{i=1}^{n} \ln \left(2 e^{\lambda x_{i}}+3 \alpha-2\right) \\
& -2 \sum_{i=1}^{n}\left\{\ln \left(e^{\lambda x_{i}}+\alpha-1\right)+\ln \left(e^{\lambda x_{i}}+2 \alpha-1\right)\right\} .
\end{aligned}
$$

It follows that the maximum-likelihood estimators (MLEs) $(\widehat{\alpha}, \widehat{\lambda})$ of $(\alpha, \lambda)$ are obtained by solving the system of equations:

$$
\frac{\partial \ell}{\partial \alpha}=0, \quad \frac{\partial \ell}{\partial \lambda}=0 .
$$

That is,

$$
\begin{gathered}
\frac{2 n}{\alpha}+\sum_{i=1}^{n} \frac{3}{2 e^{\lambda x_{i}}+3 \alpha-2}-\frac{2}{e^{\lambda x_{i}}+\alpha-1}-\frac{4}{e^{\lambda x_{i}}+2 \alpha-1}=0, \\
\frac{n}{\lambda}+n \bar{x}+\sum_{i=1}^{n} \frac{2 x_{i} e^{\lambda x_{i}}}{2 e^{\lambda x_{i}}+3 \alpha-2}-\frac{2 x_{i} e^{\lambda x_{i}}}{e^{\lambda x_{i}}+\alpha-1}-\frac{2 x_{i} e^{\lambda x_{i}}}{e^{\lambda x_{i}}+2 \alpha-1}=0 .
\end{gathered}
$$

The last system of equations does not have explicit solution and numerical techniques are required to obtain the MLEs. Alternatively, the MLEs of the parameters can be obtained by directly maximizing the log-likelihood function $\ell(\alpha, \lambda)$, using numerical optimization algorithms. For example, the nlm (nonlinear minimization) function in $\mathrm{R}$ language ( $\mathrm{R}$ Core Team 2016) or the numerical maximization function NMaximize in Mathematica software (Wolframe). 


\subsection{Asymptotic distribution of MLEs}

For interval estimation and tests of hypotheses, the asymptotic distribution of the MLE is needed. Under certain regularity conditions [16], the $\operatorname{MLE}(\widehat{\alpha}, \widehat{\lambda})$ is asymptotically bivariate normal with mean $(\alpha, \lambda)$ and variance-covariance matrix $\mathbf{J}_{n}^{-1}(\alpha, \lambda)$ where

$$
\mathbf{J}_{n}(\alpha, \lambda)=\left[\begin{array}{ll}
J_{11}(\alpha, \lambda) & J_{12}(\alpha, \lambda) \\
J_{12}(\alpha, \lambda) & J_{22}(\alpha, \lambda)
\end{array}\right]=\left[\begin{array}{cc}
-\frac{\partial^{2} \ell}{\partial \alpha^{2}} & -\frac{\partial^{2} \ell}{\partial \alpha \partial \lambda} \\
-\frac{\partial^{2} \ell}{\partial \alpha \partial \lambda} & -\frac{\partial^{2} \ell}{\partial \lambda^{2}}
\end{array}\right]
$$

is the observed information matrix with elements:

$$
\begin{aligned}
& J_{11}=\frac{2 n}{\alpha^{2}}+\sum_{i=1}^{n}\left\{\frac{9}{\left(2 e^{\lambda x_{i}}+3 \alpha-2\right)^{2}}-\frac{2}{\left(e^{\lambda x_{i}}+\alpha-1\right)^{2}}\right. \\
& J_{12}=\sum_{i=1}^{n} 2 x_{i} e^{\lambda x_{i}}\left\{\frac{8}{\left(e^{\lambda x_{i}}+2 \alpha-1\right)^{2}}\right\}, \\
& J_{22}=\frac{3}{\lambda^{2}}-\sum_{i=1}^{n} 2 x_{i}^{2} e^{\lambda x_{i}}\left\{\frac{1}{\left(e^{\lambda x_{i}}+\alpha-2\right)^{2}}\right. \\
& \left.\frac{3 \alpha-2}{\left(2 e^{\lambda x_{i}}+3 \alpha-2\right)^{2}}-\frac{2}{\left(e^{\lambda x_{i}}+\alpha-1\right)^{2}}\right\}, \\
& \left.-\frac{2 \alpha-1}{\left(e^{\lambda x_{i}}+2 \alpha-1\right)^{2}}\right\} .
\end{aligned}
$$

\subsection{Simulations study}

We perform a simulation study to investigate the finite sample behavior of the MLEs $(\widehat{\alpha}, \hat{\lambda})$ of the parameters $(\alpha, \lambda)$ and their asymptotic confidence intervals based on normality. We simulated 10,000 random samples each of size $n$ from the GoWE distribution using the quantile function, see equation (4),

$$
x_{i}=\frac{1}{\lambda} \ln \left[1+\frac{\alpha}{2}\left(\sqrt{\frac{8}{1-u_{i}}+1}-3\right)\right], \quad i=1,2, \ldots, n,
$$

where $u_{1}, u_{2}, \ldots, u_{n}$ is a random sample from the uniform distribution over the unit interval ( $n=50,100,150,200,250,300)$ from the GoWE distribution with 

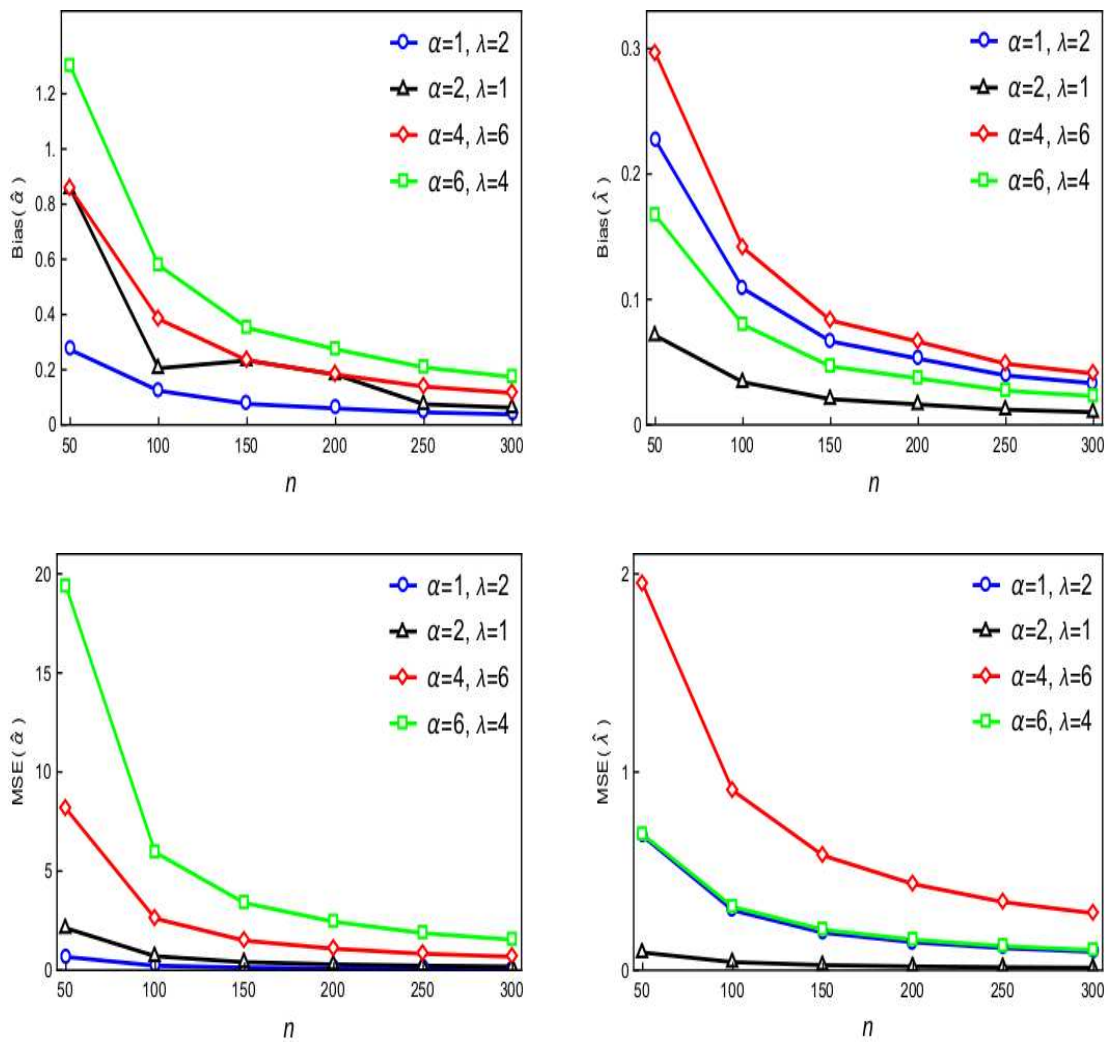

Figure 7: Biases (upper panel) and MSEs (lower panel) of the MLEs $\widehat{\alpha}$ and $\widehat{\lambda}$.

true $(\alpha, \lambda)=(1,2),(2,1),(4,6),(6,4)$. Note that the first (last) two selected values of $(\alpha, \lambda)$ represent decreasing (unimodal) shape of the p.d.f. of GoWE1 distribution. For each sample, the MLEs $(\widehat{\alpha}, \widehat{\lambda})$ were computed using the nlm (non-linear minimization) function in the R programming language (see [17]).

The evaluation of point estimation was performed based on the average bias and average mean squared error (MSE) for each sample size. The biases and MSEs of $(\widehat{\alpha}, \widehat{\lambda})$ versus $n$ are shown in Figure 7 . The biases are positive. The biases and MSEs decrease with increasing $n$.

The evaluation of interval estimation was performed based on the coverage probability (CP) of $95 \%$ confidence intervals of each parameter and the average width (AW) 95\% confidence intervals of each parameter for each sample size.

The CPs and AWs of $95 \%$ CIs of $\alpha$ and $\lambda$ versus $n$ are shown in Figure 

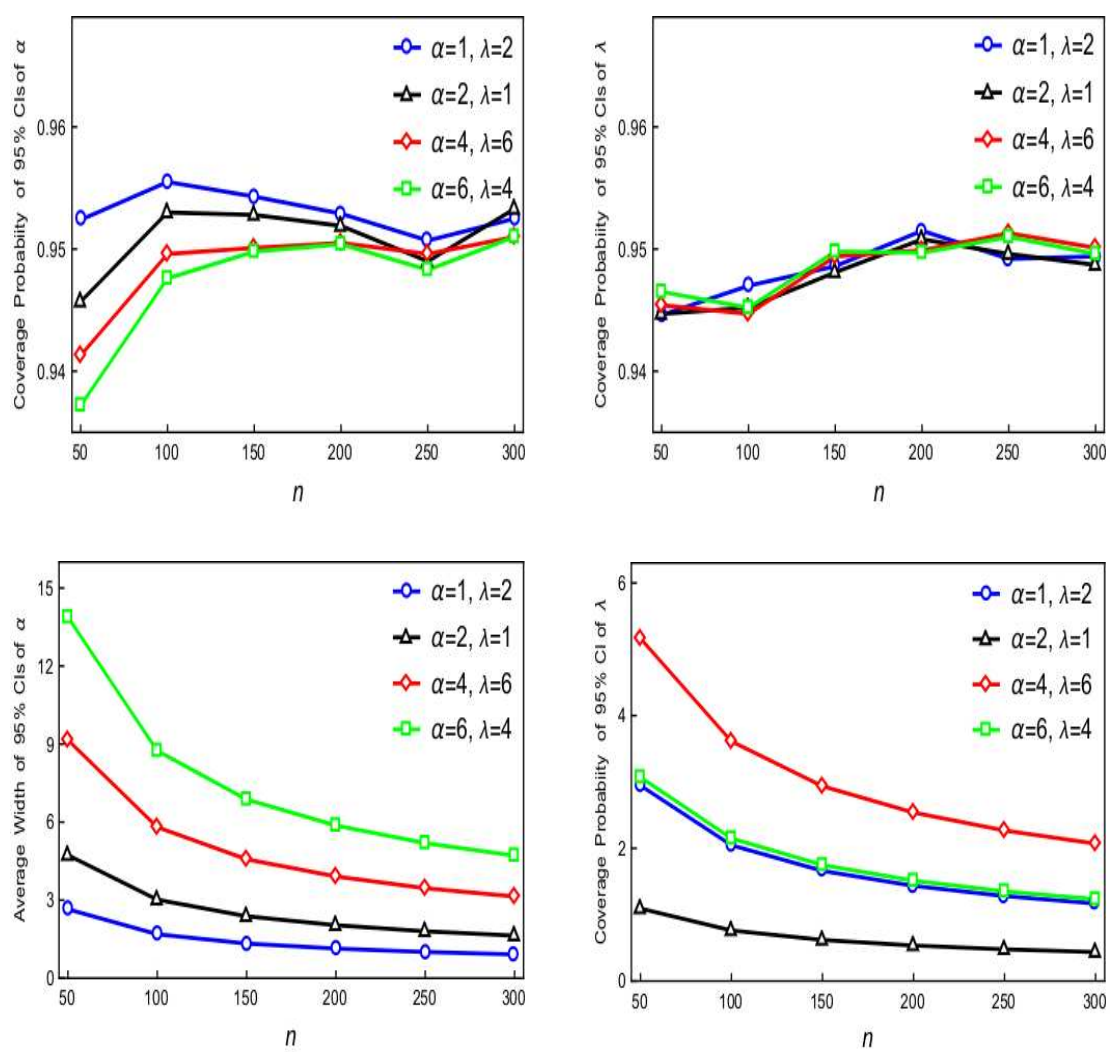

Figure 8: PCs (upper panel) and AWs (lower panel) of 95\% CIs of $\alpha$ and $\lambda$.

8. This figure show that the coverage probabilities of the confidence intervals are close to the nominal level of $95 \%$ and that the average confidence widths decrease as the sample size increases, as one would expect.

\section{Data analysis}

Here, we provide three applications of real data sets to demonstrate the flexibility and applicability of the GoWE distribution over many well known twoparameter distributions. For all the following two-parameter distributions the 
support is $x>0$ and the parameter values are $\alpha, \lambda>0$.

(1) Gamma distribution:

$$
f_{1}(x)=\frac{\lambda^{\alpha}}{\Gamma(\alpha)} x^{\alpha-1} e^{-\lambda x} .
$$

(2) Weibull distribution:

$$
f_{2}(x)=\alpha \lambda^{\alpha} x^{\alpha-1} e^{-(\lambda x)^{\alpha}} .
$$

(3) Exponentiated Exponential distribution: ([18]):

$$
f_{3}(x)=\alpha \lambda e^{-\lambda x}\left(1-e^{-\lambda x}\right)^{\alpha-1} .
$$

(4) Lomax distribution:

$$
f_{4}(x)=\alpha \lambda(1+\lambda x)^{-\alpha-1} .
$$

(5) Linear Failure Rate distribution:

$$
f_{5}(x)=(\alpha+\lambda x) e^{-\alpha x-\frac{\lambda}{2} x^{2}} .
$$

(6) Exponentiated Rayleigh (Burr X) distribution: ([4]):

$$
f_{6}(x)=2 \alpha \lambda^{2} x e^{-(\lambda x)^{2}}\left(1-e^{-(\lambda x)^{2}}\right)^{\alpha-1} .
$$

(7) Exponential Power distribution ([5]):

$$
f_{7}(x)=\alpha \lambda^{\alpha} x^{\alpha-1} \exp \left[(\lambda x)^{\alpha}-\left(e^{(\lambda x)^{\alpha}}-1\right)\right] .
$$

(8) Double Exponential Power distribution ([6]):

$$
f_{8}(x)=\alpha \lambda^{\alpha} x^{\alpha-1} \exp \left\{(\lambda x)^{\alpha}+e^{(\lambda x)^{\alpha}}-\exp \left[e^{(\lambda x)^{\alpha}}-1\right]\right\} .
$$

(9) Chen distribution ([7]):

$$
f_{9}(x)=\alpha \lambda x^{\alpha-1} \exp \left[x^{\alpha}-\lambda\left(e^{x^{\alpha}}-1\right)\right] .
$$

(10) Generalized Lindley distribution ([9]):

$$
f_{10}(x)=\frac{\alpha \lambda^{2}}{1+\lambda}(1+x) e^{-\lambda x}\left(1-\frac{1+\lambda+\lambda x}{1+\lambda} e^{-\lambda x}\right)^{\alpha-1} .
$$



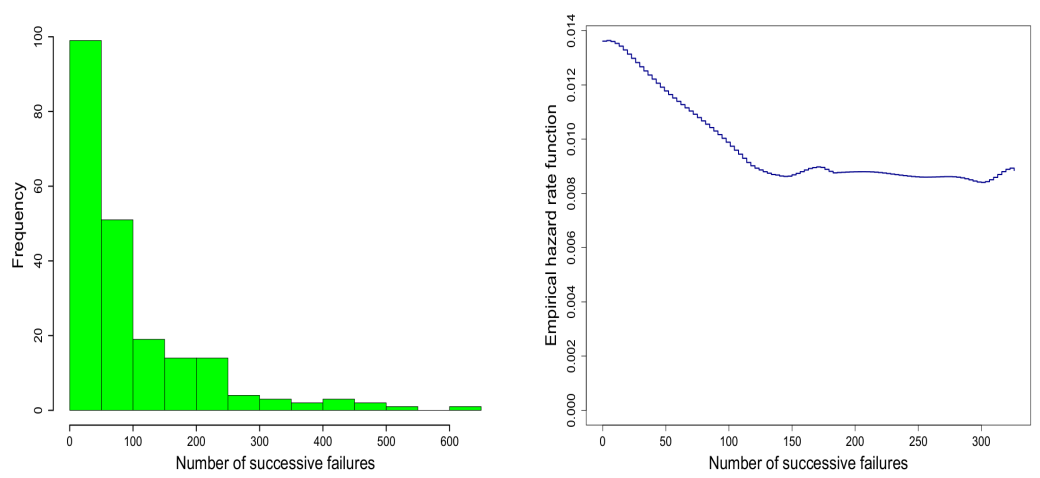

Figure 9: Histogram and e.h.r.f. of data set 1.

Note that the above distributions (1)-(5) have monotone h.r.f. and distributions (6)-(10) have bathtub h.r.f.

Data set 1. This data set consists of 213 observations on the number of successive failures of the air conditioning system of a fleet of 13 Boeing 720 jet airplanes, see [19]. Its histogram and empirical hazard rate function (e.h.r.f.) are shown in Figure 9. The e.h.r.f. shows decreasing shape.

Table 1 shows the estimates, their standard error (S.E.) of the parameters, the estimated log-liklihood and two goodness-of-fit tests for six different distributions which allow for decreasing h.r.f.

Table 1: MLEs (S.E.), estimated log-likelihood, Anderson-Darling (AD) and Cramér-von Mises (CvM) goodness-of-fit tests for data set 1 .

\begin{tabular}{|l|cc|c|cc|}
\hline Distribution & $\begin{array}{c}\widehat{\alpha} \\
\text { S.E. })\end{array}$ & $\begin{array}{c}\hat{\lambda} \\
\text { S.E. })\end{array}$ & $\ell(\widehat{\alpha}, \widehat{\lambda})$ & $\begin{array}{c}\text { AD } \\
(p \text {-value })\end{array}$ & $\begin{array}{c}\text { CvM } \\
(p \text {-value })\end{array}$ \\
\hline \hline Gamma & 0.922 & 0.010 & -1178.291 & 1.124 & 0.199 \\
& $(0.078)$ & $(0.001)$ & & $(0.298)$ & $(0.269)$ \\
\hline Weibull & 0.925 & 0.011 & -1177.585 & 0.825 & 0.128 \\
& $(0.048)$ & $(0.001)$ & & $(0.464)$ & $(0.466)$ \\
\hline Exponentiated & 0.927 & 0.010 & -1178.402 & 1.409 & 0.275 \\
Exponential & $(0.083)$ & $(0.001)$ & & $(0.200)$ & $(0.159)$ \\
\hline Lomax & 6.075 & 0.002 & -1176.418 & 0.467 & 0.061 \\
& $(3.196)$ & $(0.001)$ & & $(0.781)$ & $(0.811)$ \\
\hline Generalized & 0.462 & 0.013 & -1181.698 & 4.197 & 0.762 \\
Lindley & $(0.041)$ & $(0.001)$ & & $(0.007)$ & $(0.009)$ \\
\hline GoWE & 0.377 & 0.003 & -1175.950 & 0.461 & 0.055 \\
& $(0.135)$ & $(0.001)$ & & $(0.786)$ & $(0.846)$ \\
\hline
\end{tabular}



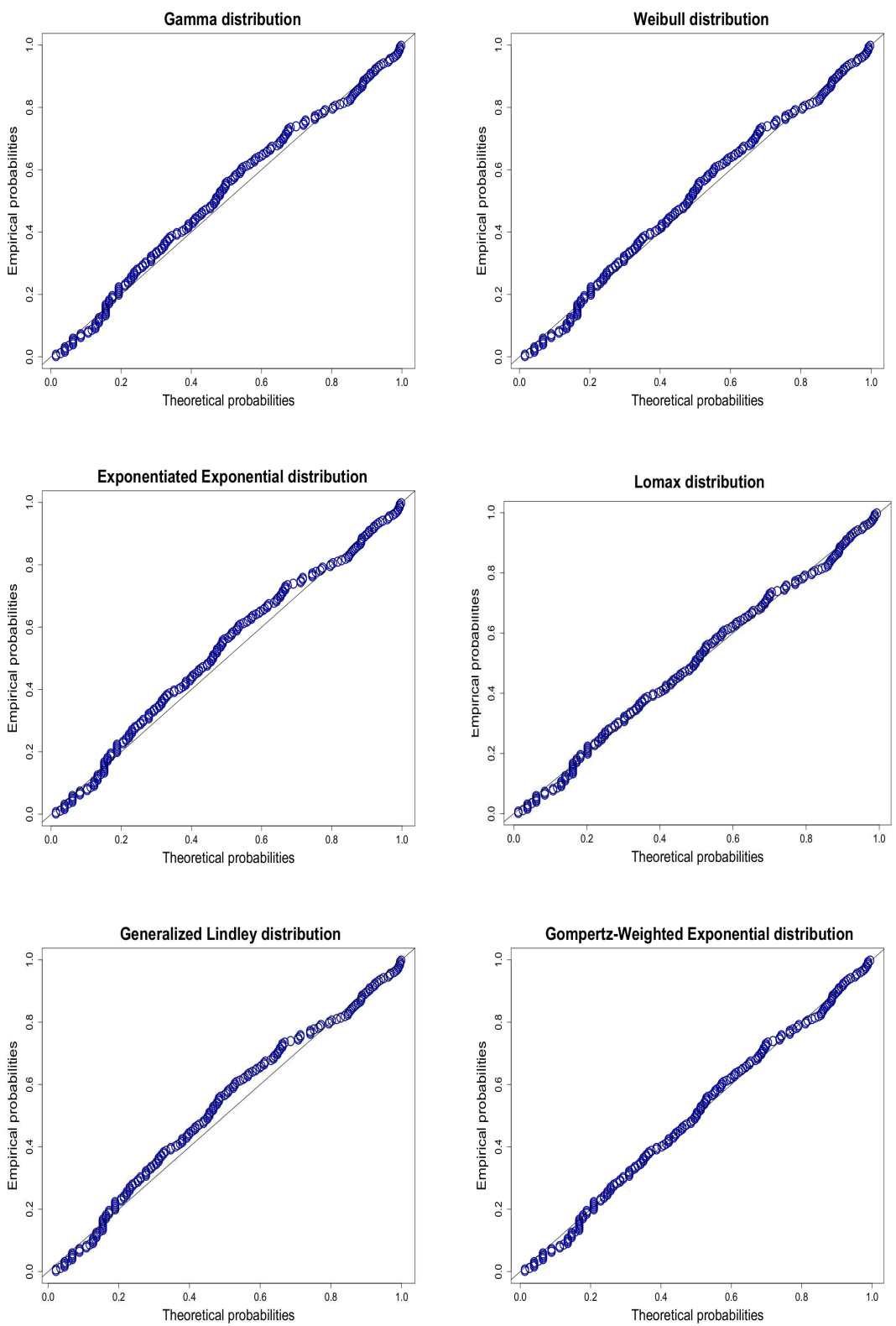

Figure 10: P-P plots of competing distributions for data set 1. 
Data set 2. This data set represents the lowest 7-day average flows (in meter cube per second) at gauging station La Parota during 1963-1999, see [20]. Its histogram and e.h.r.f. are shown in Figure 11. The e.h.r.f. shows increasing shape.
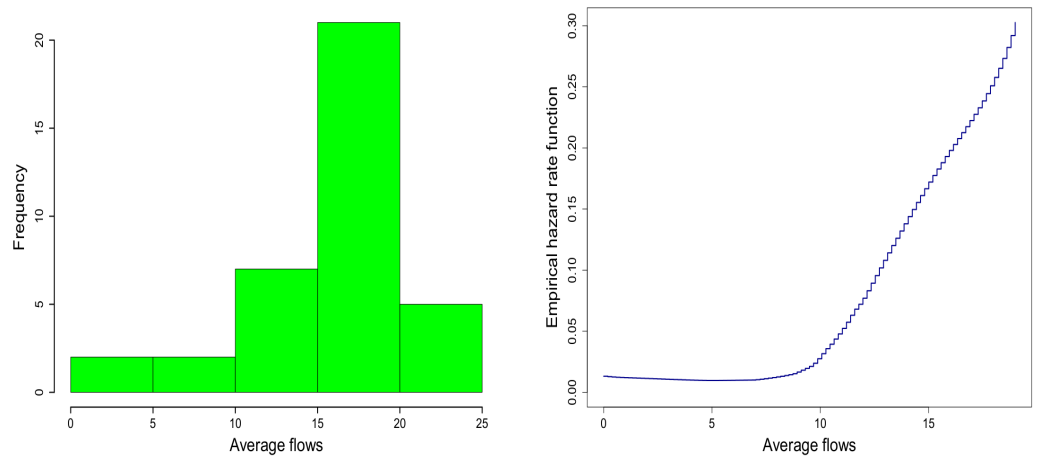

Figure 11: Histogram and e.h.r.f. of data set 2.

Table 2 shows the estimates, their standard error (S.E.) of the parameters, the estimated log-liklihood and two goodness-of-fit tests for six different distributions which allow for increasing h.r.f.

Table 2: MLEs (S.E.), estimated log-likelihood, Anderson-Darling (AD) and Cramér-von Mises (CvM) goodness-of-fit tests for data set 2.

\begin{tabular}{|l|cc|c|cc|}
\hline & $\widehat{\alpha}$ & $\widehat{\lambda}$ & & AD & CvM \\
Distribution & $($ S.E. $)$ & $($ S.E. $)$ & $\ell(\widehat{\alpha}, \widehat{\lambda})$ & $(p$-value $)$ & $(p$-value $)$ \\
\hline \hline Gamma & 4.241 & 0.267 & -125.055 & 4.125 & 0.744 \\
& $(0.950)$ & $(0.063)$ & & $(0.008)$ & $(0.010)$ \\
\hline Weibull & 3.664 & 0.058 & -114.202 & 1.658 & 0.246 \\
& $(0.518)$ & $(0.003)$ & & $(0.143)$ & $(0.193)$ \\
\hline Exponentiated & 3.803 & 0.123 & -127.802 & 4.783 & 0.887 \\
Exponential & $(0.940)$ & $(0.017)$ & & $(0.004)$ & $(0.004)$ \\
\hline Linear Failure Rate & 0.002 & 0.007 & -121.085 & 5.199 & 1.076 \\
& $(0.004)$ & $(0.001)$ & & $(0.002)$ & $(0.001)$ \\
\hline Generalized & 2.491 & 0.174 & -124.470 & 4.197 & 0.762 \\
Lindley & $(0.628)$ & $(0.022)$ & & $(0.007)$ & $(0.009)$ \\
\hline Gompertz-Weighted & 702.216 & 0.364 & -105.273 & 0.407 & 0.037 \\
Exponential & $(645.515)$ & $(0.051)$ & & $(0.841)$ & $(0.949)$ \\
\hline
\end{tabular}



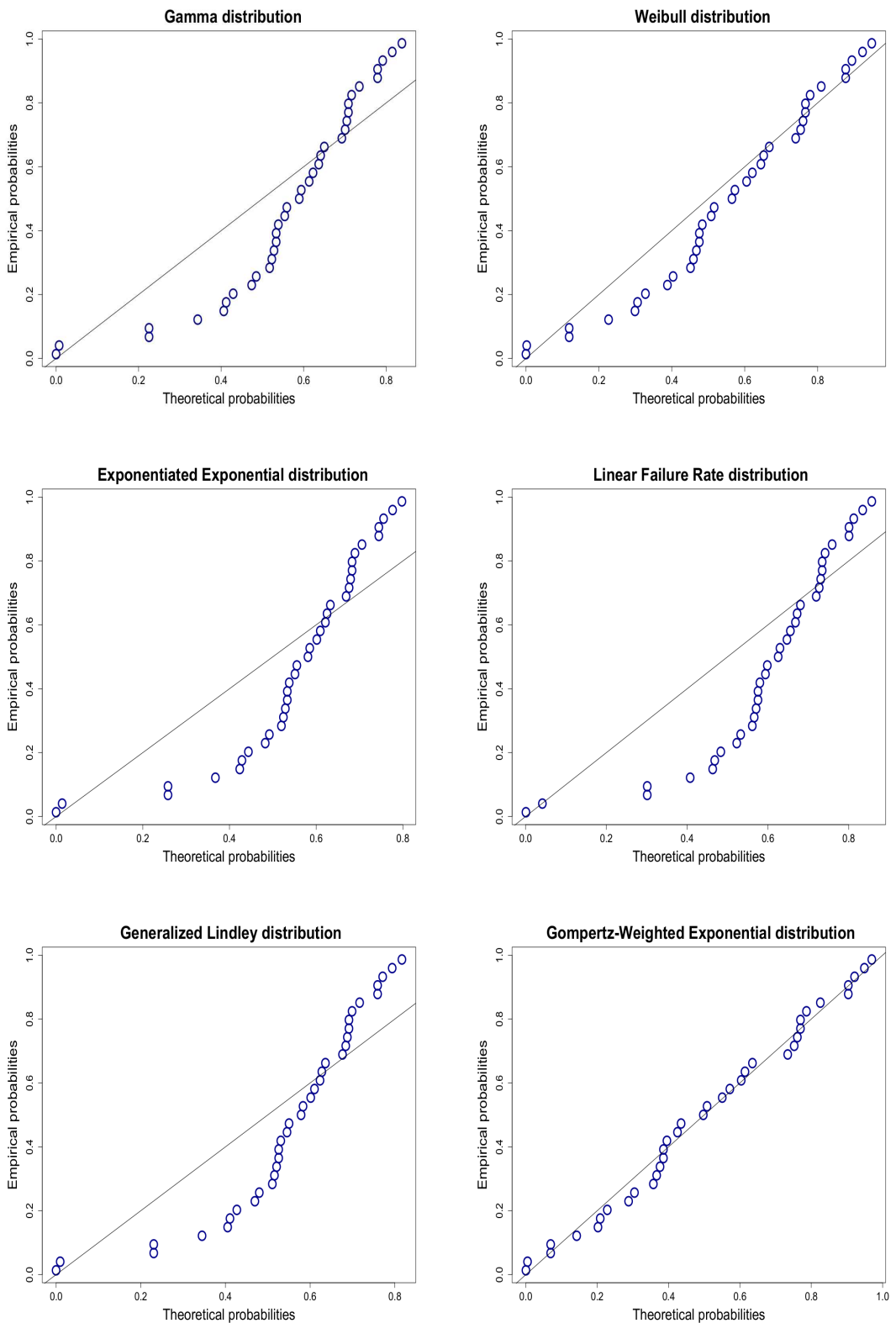

Figure 12: P-P plots of competing distributions for data set 2. 
Data set 3. This data set represents 60 times between failures (in 1000's of hours) of a 200-ton rear dump truck. Its histogram and e.h.r.f. are shown in Figure 13. The e.h.r.f. shows bathtub shape.
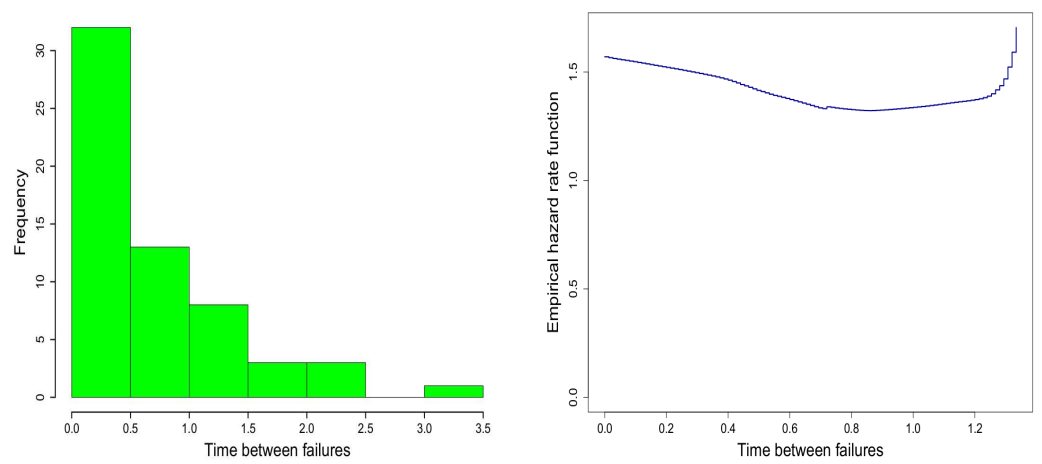

Figure 13: Histogram and e.h.r.f. of data set 3.

Table 3 shows the estimates, their standard error (S.E.) of the parameters, the estimated log-liklihood and two goodness-of-fit tests for six different distributions which allow for bathtub hazard rate function.

Table 3: MLEs (S.E.), estimated log-likelihood, Anderson-Darling (AD) and Cramér-von Mises (CvM) goodness-of-fit tests for data set 3 .

\begin{tabular}{|c|c|c|c|c|c|}
\hline Distribution & $\begin{array}{c}\widehat{\alpha} \\
\text { (S.E.) }\end{array}$ & $\begin{array}{c}\widehat{\lambda} \\
(\text { S.E. })\end{array}$ & $\ell(\widehat{\alpha}, \widehat{\lambda})$ & $\begin{array}{c}\mathrm{AD} \\
(p \text {-value })\end{array}$ & $\begin{array}{c}\text { CvM } \\
(p \text {-value })\end{array}$ \\
\hline Burr X & $\begin{array}{c}0.360 \\
(0.053)\end{array}$ & $\begin{array}{c}0.723 \\
(0.085)\end{array}$ & -37.877 & $\begin{array}{c}0.611 \\
(0.637)\end{array}$ & $\begin{array}{c}0.116 \\
(0.513)\end{array}$ \\
\hline Exponential Power & $\begin{array}{c}0.719 \\
(0.076)\end{array}$ & $\begin{array}{c}0.793 \\
(0.088)\end{array}$ & -37.483 & $\begin{array}{c}0.422 \\
(0.826)\end{array}$ & $\begin{array}{c}0.069 \\
(0.757)\end{array}$ \\
\hline $\begin{array}{l}\text { Double } \\
\text { Exponential Power }\end{array}$ & $\begin{array}{c}0.549 \\
(0.062)\end{array}$ & $\begin{array}{c}0.384 \\
(0.039)\end{array}$ & -39.320 & $\begin{array}{c}0.812 \\
(0.472)\end{array}$ & $\begin{array}{c}0.134 \\
(0.442)\end{array}$ \\
\hline Chen & $\begin{array}{c}0.675 \\
(0.065)\end{array}$ & $\begin{array}{c}0.783 \\
(0.103)\end{array}$ & -37.770 & $\begin{array}{c}0.438 \\
(0.810)\end{array}$ & $\begin{array}{c}0.065 \\
(0.785)\end{array}$ \\
\hline $\begin{array}{l}\text { Generalized } \\
\text { Lindley }\end{array}$ & $\begin{array}{c}0.927 \\
(0.160)\end{array}$ & $\begin{array}{c}1.898 \\
(0.270)\end{array}$ & -36.564 & $\begin{array}{c}0.190 \\
(0.993)\end{array}$ & $\begin{array}{c}0.028 \\
(0.984)\end{array}$ \\
\hline $\begin{array}{l}\text { Gompertz-Weighted } \\
\text { Exponential }\end{array}$ & $\begin{array}{c}0.764 \\
(0.410)\end{array}$ & $\begin{array}{c}0.764 \\
(0.285)\end{array}$ & -36.447 & $\begin{array}{c}0.146 \\
(0.999)\end{array}$ & $\begin{array}{c}0.019 \\
(0.998)\end{array}$ \\
\hline
\end{tabular}

For all three data sets, Tables 1-3 show that the GoWE distribution has the highest estimated log-likelihood, the smallest test statistic and largest $p$ value of the two goodness-of-fit tests. Thus, we can conclude that the GoWE distribution provides the best fit for thee data sets. This conclusion is also supported by the Probability-Probability (P-P) plots in Figures 10-14. 

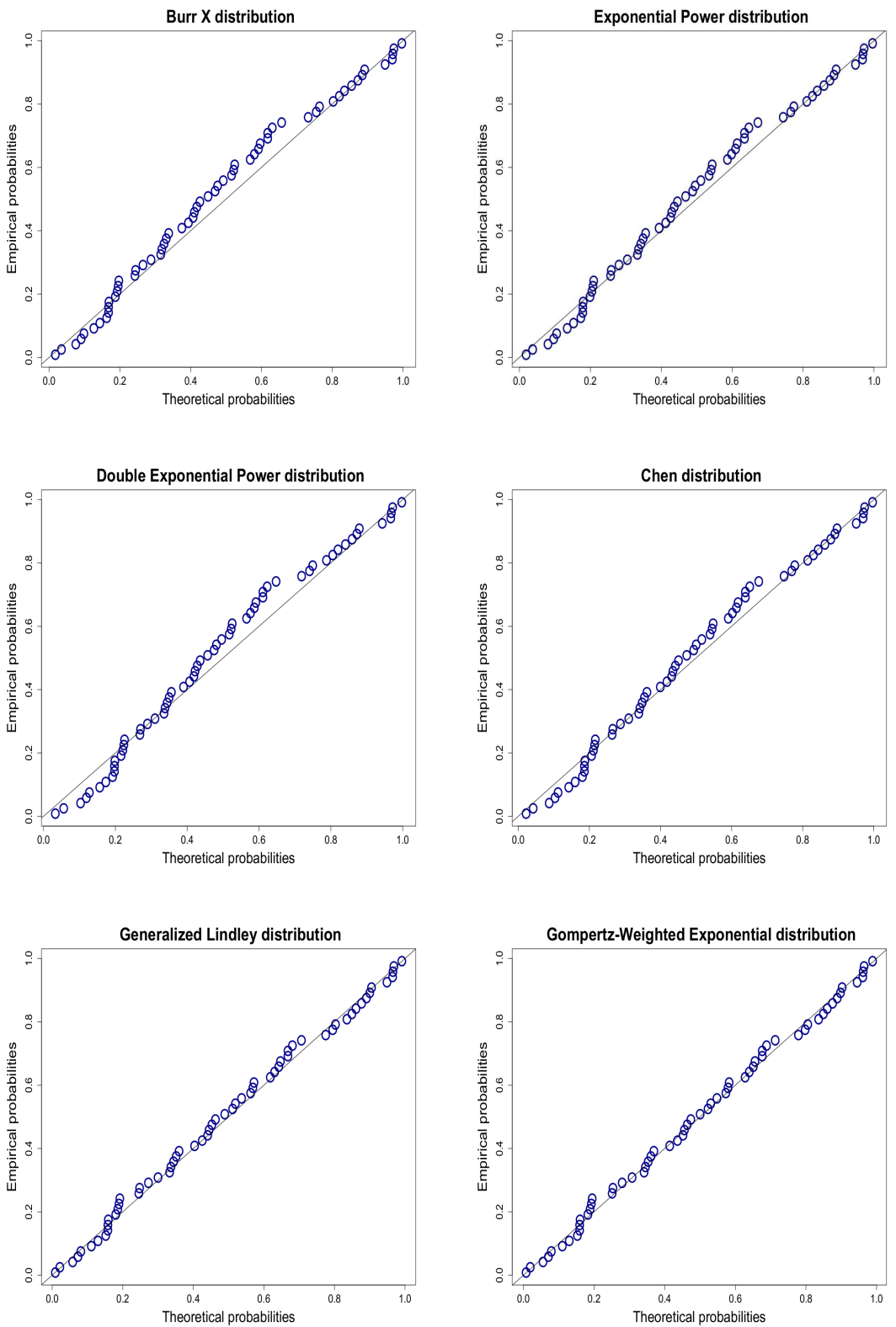

Figure 14: P-P plots of competing distributions for data set 3. 


\section{References}

[1] S. Rajarshi, M. Rajarshi, Bathtub distributions: A review, Communications in Statistics-Theory and Methods, 17 (1988), 2597-2621.

[2] C. Lai, M. Xie, D.N.P. Murthy, Bathtub-shaped failure rate life distributions, In: Handbook of Statistics, 20, Elsevier, Amsterdam (2001), 69-104.

[3] S. Nadarajah, Bathtub-shaped failure rate functions, Quality and Quantity, 43 (2009), 855-863.

[4] I.W. Burr, Cumulative frequency functions, Annals of Mathematical Statistics, 13 (1942), 215-232.

[5] R.M. Smith, L.J. Bain, An exponential power life-testing distribution, Communications in Statistics-Theory and Methods, 4 (1975), 469-481.

[6] S. Paranjpe, M.B. Rajarshi, Modelling non-monotonic survivorship data with bathtub distributions, Ecology, 67 (1986), 1693-1695.

[7] Z. Chen, A new two-parameter lifetime distribution with bathtub shape or increasing failure rate function, Statistics and Probability Letters, 49 (2000), 155-161.

[8] M.E. Ghitany, F. Alqallaf, D.K. Al-Mutairi, H.A. Husain, A two-parameter weighted Lindley distribution and its applications to survival data, Mathematics and Computers in Simulation, 81 (2011), 1190-1201.

[9] S. Nadarajah, H.S. Bakouch, R. Tahmasbi, A generalized Lindley distribution, Sankhya B, 73 (2011), 331-359.

[10] A. Marshall, I. Olkin, A new method for adding a parameter to a family of distributions with application to the exponential and Weibull families, Biometrika, 84 (1997), 641-652.

[11] M. Bryson, M.M. Siddiqui, Some criteria for aging, Journal of the American Statistical Association, 64 (1969), 1472-1483.

[12] R.C. Gupta, H. Akman, Mean residual life function for certain types of non-monotonic ageing, Communications in Statistics-Stochastic Models, 11 (1995), 219-225.

[13] L. Lewin, Polylogarithms and associated functions, North Holland, New York (1981). 
[14] B. Arnold, N. Balakrishnan, H. Nagaraja, A First Course in Order Statistics, Wiley, New York (1992).

[15] M. Shaked, J. Shanthikumar, Stochastic Orders, Springer, New York (2007).

[16] D. Cox, D. Hinkley, Theoretical Statistics, Chapman and Hall, New York (1974).

[17] R Core Team, R: A Language and Environment for Statistical Computing, R Foundation for Statistical Computing, Vienna, Austria (2018).

[18] P. Gupta, R.C. Gupta, R.D. Gupta, Modeling failure time data by Lehman alternatives, Communications in Statistics-Theory and Methods, 27 (1998), 887-904.

[19] F. Proschan, Theoretical explanation of observed decreasing failure rate, Technometrics, 5 (1963), 375-383.

[20] C. Escalante-Sandoval, Mixed distributions in low frequency analysis, Ingeniera, investigacin y tecnologa, 10 (2009), 247-253. 